\title{
Revisiting the phase transition of magnetite under pressure
}

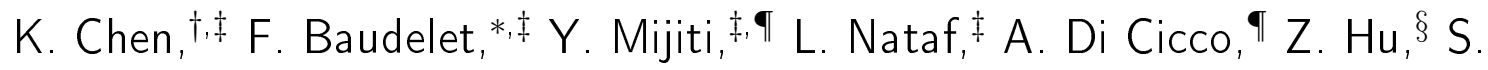 \\ Agrestini, ${ }^{\S}$ A. C. Komarek, ${ }^{\S}$ M. Sougrati, I J. Haines, I J. Rouquette, ${ }^{\prime}$ Q. Kong, ${ }^{\ddagger}$ \\ and T-C. Weng ${ }^{*, \dagger}$ \\ $\dagger$ Center for High Pressure Science \& Technology Advanced Research, 1690 Cailun Rd, \\ 201203 Shanghai, China \\ $\ddagger$ Synchrotron SOLEIL, L'Orme des Merisiers, Saint-Aubin, BP 48, 91192 Gif-sur-Yvette \\ Cedex, France \\ \Physics Division, School of Science and Technology, University of Camerino, I-62032 \\ Camerino (MC), Italy \\ §Max Planck Institute for Chemical Physics of Solids, Nöthnizer Strasse 40, 01187 \\ Dresden, Germany \\ \|ICGM, CNRS, Universite de Montpellier, ENSCM, Montpellier, France \\ E-mail: francois.baudelet@synchrotron-soleil.fr; tsuchien.weng@hpstar.ac.cn
}

\begin{abstract}
Using a highly stoichiometric magnetite, the pressure-induced phase transitions of $\mathrm{Fe}_{3} \mathrm{O}_{4}$ have been revisited here by performing Fe K-edge x-ray absorption and magnetic circular dichroism measurements up to $\mathrm{P}=65 \mathrm{GPa}$ at room temperature and $71 \mathrm{GPa}$ at $20 \mathrm{~K}$. We have observed a structure transition at around $27 \mathrm{GPa}$ from magnetite to a high pressure phase $h-\mathrm{Fe}_{3} \mathrm{O}_{4}$ with the loss of the net ordered magnetic moments for both temperature. The orthorhombic $\mathrm{CaTi}_{2} \mathrm{O}_{4}$-type (Bbmm) structure of the high
\end{abstract}


pressure phase $h$ - $\mathrm{Fe}_{3} \mathrm{O}_{4}$ has been determined by combining experimental studies and theoretical simulations of the Fe K-edge XANES. The long time discussed pressure induced spin crossover and inverse to normal spinel structure transitions in $\mathrm{Fe}_{3} \mathrm{O}_{4}$ can be deterministically excluded from present study up to $\sim 65 \mathrm{GPa}$.

Being the first discovered natural magnet, magnetite $\left(\mathrm{Fe}_{3} \mathrm{O}_{4}\right)$ has a cubic inverse spinel structure $(\mathrm{Fd} \overline{3} \mathrm{~m})$ with the chemical formula of $\mathrm{AB}_{2} \mathrm{O}_{4}$. The A-site ions are tetrahedrally $(\mathrm{Td})$ coordinated, with the nominal $\mathrm{Fe}^{3+}(\mathrm{S}=-5 / 2)$ configuration, while B-site ions are octahedrally $(\mathrm{Oh})$ coordinated with equal distribution of $\mathrm{Fe}^{2+}(\mathrm{S}=2)$ and $\mathrm{Fe}^{3+}(\mathrm{S}=5 / 2)$. The opposite spin alignment of Fe ions on A- and B-sites leads to ferrimagnetism, resulting in a net moment of $\mathrm{M}_{\text {net }}=+4 \mu \mathrm{B}$ per formula unit, as compared to the experimental value of $4.07 \mu \mathrm{B} .{ }^{1}$ The so called Verwey transition ${ }^{2-4}$ in magnetite, described as an abrupt hundred-fold increase of electrical resistivity (metal-insulator transition) with temperature decreasing across $\mathrm{T}_{\mathrm{V}} \sim$ $124 \mathrm{~K}$, has been associated to charge ordering and three-site distortions of Fe ions ${ }^{5,6}$ with a gap opening in the electronic band structure. ${ }^{7}$

Before entering the high pressure phase around $\mathrm{P}=30 \mathrm{GPa}\left(h-\mathrm{Fe}_{3} \mathrm{O}_{4}\right),{ }^{8-13}$ a number of electronic, magnetic, and structural transitions in magnetite have been reported around 8 GPa, including HS to IS transition, ${ }^{14}$ the pressure-tuned ideal inverse-spinel structure,${ }^{15}$ the inverse to normal spinal, ${ }^{7,16-19}$ as well as the occurrence of abnormal elastic and vibrational behaviors. ${ }^{20}$ However, the scenario of inverse to normal spinal was not supported by neutron powder diffraction, ${ }^{21}$ single-crystal x-ray diffraction ${ }^{22}$ and Fe K-edge x-ray magnetic circular dichroism spectroscopy (XMCD). ${ }^{14,23,24}$ The spin transition from high spin (HS) to intermediate spin (IS) state of B-site $\mathrm{Fe}^{2+}$ ions at around $12 \mathrm{GPa},{ }^{14}$ was later ruled out using the same Fe K-edge XMCD technique. ${ }^{24}$ From first-principles density-functional theory, ${ }^{25}$ the net magnetic moment of $\mathrm{Fe}_{3} \mathrm{O}_{4}$ should show an abrupt drop when entering into the high-pressure phase at $\mathrm{P}=30 \mathrm{GPa}$ with $\mathrm{HS} \rightarrow \mathrm{IS}$ and the net magnetization will be recoverd at around $\mathrm{P} \sim$ $70 \mathrm{GPa}$ with the post-spinel structural phase (Bbmm). ${ }^{12}$ Another ab initio study concluded 
that $\mathrm{Fe}_{3} \mathrm{O}_{4}$ had no spin transition in $\mathrm{Fe}^{2+}$ up to $45 \mathrm{GPa},{ }^{26}$ confirmed by the $\mathrm{Fe} \mathrm{K}_{\beta} \mathrm{X}$-ray emission spectra of single-crystal magnetite at high pressures. ${ }^{20}$ Besides, Xu et al., showed an incipient metallic behavior in $\mathrm{Fe}_{3} \mathrm{O}_{4}$ coupled to a magnetic moment collapse at a higher pressure of $50 \mathrm{GPa},{ }^{27}$ while Muramatsu et al., claimed the metal-insulator-semiconductor transition at $\mathrm{P}=\sim 30$ and $70 \mathrm{GPa}$ respectively. ${ }^{28}$

The existing results differ from each other significantly, which may due to the oxygen stoichiometry or the inhomogeneity of the magnetite used in those studies, or the nonhydrostatic conditions with different pressure transmitting medium (PTM). Thus the phase transition of magnetite under pressure requires further careful investigation. In this paper, we present experimental X-ray absorption near edge spectra (XANES) and XMCD spectra of a highly stoichiometric magnetite at Fe K-edge up to $65 \mathrm{GPa}$, together with the simulated XANES spectra of magnetite $(\mathrm{Fd} \overline{3} \mathrm{~m})$ and $\mathrm{CaTi}_{2} \mathrm{O}_{4}$-type structure $(\mathrm{Bbmm}) h$-Fe $\mathrm{O}_{4}$. For pressures $\mathrm{P}>33 \mathrm{GPa}, \mathrm{Fe}_{3} \mathrm{O}_{4}$ loses its ferrimagnetism and enters the $h-\mathrm{Fe}_{3} \mathrm{O}_{4}$ phase. The pressure induced charge transfer from octahedral B-site $\mathrm{Fe}^{2+}$ to bicapped trigonal A-site $\mathrm{Fe}^{3+}$, and the HS to IS or LS transition are not observed up to $65 \mathrm{GPa}$ in $h-\mathrm{Fe}_{3} \mathrm{O}_{4}$ in present study.

High-pressure XANES and XMCD measurements at the Fe K-edge at T=20 and 300K, have been performed at ODE beamline at synchrotron SOLEIL, France. ${ }^{29}$ Magnetite of micrometer-sized powders ground from a high-quality single crystal with $99.999 \%$ purity, together with the pressure transmitting medium of silicon oil, $\mathrm{O}_{2}$ or Ne gas, were subject to high pressure up to $71 \mathrm{GPa}$ in diamond-anvil cell (DAC). The sample qualities, including the stoichiometry and the homogeneity of the single crystal were checked by careful resistivity measurement, which has an extremely sharp Verwey transition at 124K (see Fig. S1 in Supplementary). Pressures were calibrated based on the pressure induced Ruby fluorescence spectra shift. XANES were collected at the Fe K-edge $(7112 \mathrm{eV})$ with a fixed helicity with magnetic field $\mathrm{H}=0$. XMCD was obtained through the deviation of XANES under a magnetic field of $\mathrm{H}=1.3 \mathrm{~T}$ applied parallel or antiparallel to the beam helicity. Fe K-edge XANES 

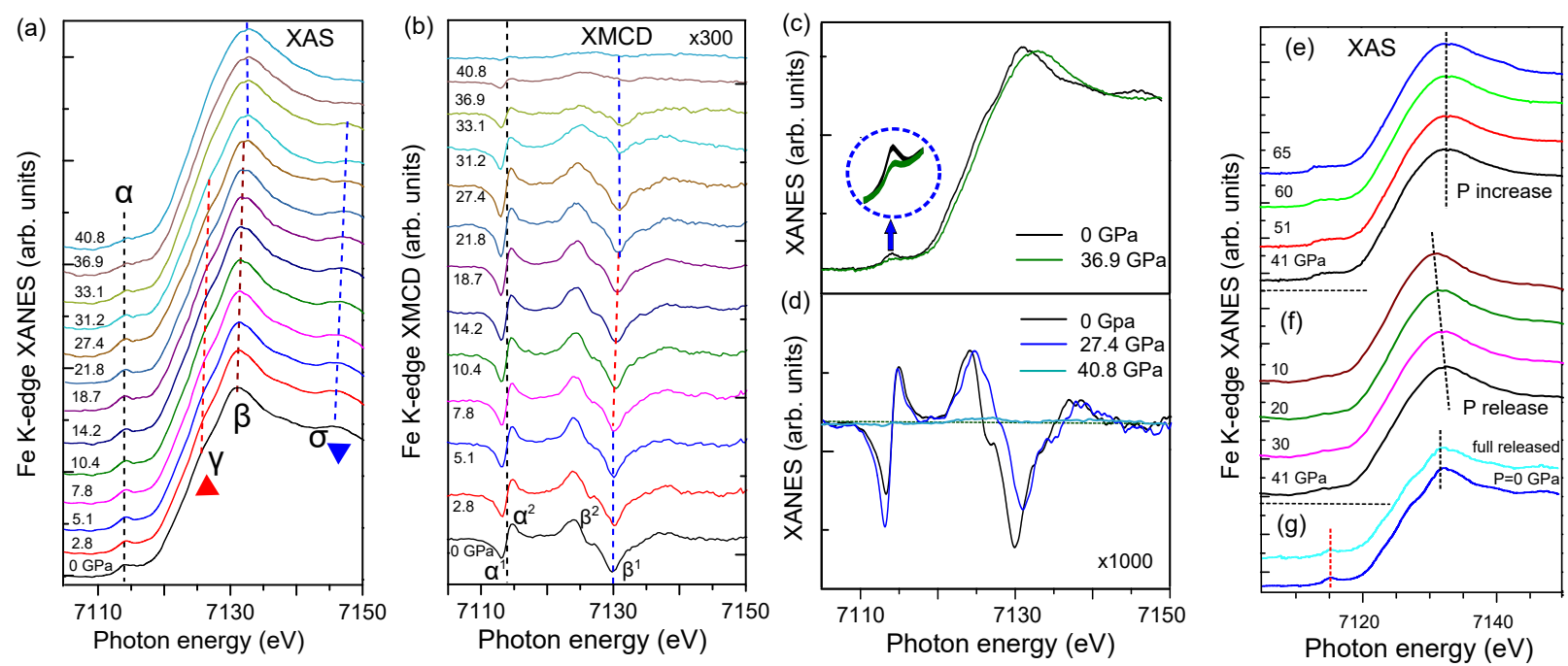

Figure 1: (a)The normalized XANES collected at RT from 0 to $40.8 \mathrm{GPa}$ and (b) the corresponding normalized XMCD. The pre-peak positions of XANES and XMCD, marked as $\alpha$ and $\alpha^{1}-\alpha^{2}$, keep constants with pressure increasing while the energy of the main peak, marked as $\beta$ and $\beta^{1}-\beta^{2}$ gradually shift to higher energy and saturated at $\sim 27.4 \mathrm{GPa}$. (c)XANES spectra of $\mathrm{Fe}_{3} \mathrm{O}_{4}$ at $\mathrm{P}=0$ and $37 \mathrm{GPa}$ and (d) XMCD spectra of $\mathrm{Fe}_{3} \mathrm{O}_{4}$ at $\mathrm{P}=0,27.4$ and $41 \mathrm{GPa}$. XANES of $h-\mathrm{Fe}_{3} \mathrm{O}_{4}$ (e) from 40 to $65 \mathrm{GPa}$ and (f) released from $40 \mathrm{GPa}$ to $10 \mathrm{GPa}$. (g) The fully recovered XANES spectrum profile when $\mathrm{Fe}_{3} \mathrm{O}_{4}$ is released to ambient pressure from $40 \mathrm{GPa}$.

simulations for magnetite $(\mathrm{Fd} \overline{3} \mathrm{~m})$ and high pressure $(\mathrm{Bbmm})$ phases of $h-\mathrm{Fe}_{3} \mathrm{O}_{4}$ have been performed by first principle calculations based on full multiple scattering theory using the MXAN code. ${ }^{30}$

The normalized Fe K-edge XANES and XMCD spectra of $\mathrm{Fe}_{3} \mathrm{O}_{4}$ from $\mathrm{P}=0$ to 65 and back to $0 \mathrm{GPa}$ at room temperature are shown in Figure 1. The XANES spectrum consists of a main peak at $7132 \mathrm{eV}$, labeled as $\beta$, and a pre-edge peak at $7114 \mathrm{eV}$, labeled as $\alpha$, which are generally assigned to $1 s-4 p$ dipole transition and $1 s-3 d$ quadruple transition due to $3 d / 4 p$ hybridization, respectively. Pre-edge peak $\alpha$ is mainly originated from $\mathrm{Fe}^{3+}$ at the tetrahedral sites. ${ }^{14,24}$ The energy position of peak $\alpha\left(\mathrm{E}_{\alpha}\right)$ does not change with pressure up to $65 \mathrm{GPa}$, however, its intensity starts to decrease at $\mathrm{P} \sim 27.4 \mathrm{GPa}$ and reaches the minimal value at $\mathrm{P} \sim 35 \mathrm{GPa}$. The main peak $\beta$ does not show any visible change of line-shape under pressure below 27.4 GPa except for a slight increase in the white line intensity and a gradual/continual shift to higher energy. Above $27.4 \mathrm{GPa}$ the spectral profile of the main 
peak $\beta$ shows a sharp change: it moves to higher energy as shown in Fig. 1c, where we show a comparison of spectra taken at $\mathrm{P}=0$ and $36.9 \mathrm{GPa}$ and at the same time the weak peaks at 7126 and $7145 \mathrm{eV}$, labeled in Fig.1 (a) as $\gamma$ and $\sigma$ respectively, gradually disappear.

Fig. $1 \mathrm{~b}$ reports the corresponding XMCD spectra as a function of external pressure up to 40.5 GPa. It is generally accepted that the XMCD signal at the $3 \mathrm{~d}$ transition metal K-edge arises from spin polarization of $4 \mathrm{p}$ states, which is originated from on-site and intra-site exchange interactions with polarized $3 \mathrm{~d}$ states, the so called $p-d$ hybridization. ${ }^{31}$ Therefore the size of XMCD signal is expected to be proportional to the net magnetic moment of $3 \mathrm{~d}$ states. Peak $\alpha(\beta)$ in the XMCD spectra of Fe K-edge is split into two components having opposite sign and labeled as $\alpha^{1}$ and $\alpha^{2}\left(\beta^{2}\right.$ and $\left.\beta^{1}\right)$. The XMCD signal of $\alpha^{1}$ and $\alpha^{2}$ does not exhibit clear change until $27.4 \mathrm{GPa}$ followed by a rapid decreasing to zero at $41 \mathrm{GPa}$ as shown in Fig.1b and Fig.3c. Here $H_{\alpha^{1}-\alpha^{2}}$ is sum of absolute value of the height of XMCD signal $\alpha^{1}$ and $\alpha^{2}$. Similar pressure induced change of XMCD signal of $\beta^{1}$ and $\beta^{2}$ is shown in Fig.3d. From 0 to $27.4 \mathrm{GPa}$, the $H_{\beta^{1}-\beta^{2}}$ decreases only slightly. Above 27.4GPa it decreases quickly to zero at $40 \mathrm{GPa}$. The changes of the XMCD spectra are shown by the data recorded at $\mathrm{P}=0,27.4$ and $41 \mathrm{GPa}$ and reported in Fig. 1d. The XANES, including the line shape remains unchanged with pressure increased from 41 to 65 GPa (Fig.1e), or reduced from 41 to $10 \mathrm{GPa}$ (Fig.1f). The XANES of $\mathrm{Fe}_{3} \mathrm{O}_{4}$ gets recovered when the pressure is totally released (Fig. 1g).

Fe K-edge XANES has been proved to be powerful tool for the precise structural characterization of transition metal oxides, including the chemical and electronic properties. For an inverse to normal spinel structure transition, the pre-edge $\alpha$ is sensitive to the valence state and a roughly $2 \mathrm{eV}$ shift to lower energy is expected from $\mathrm{Fe}^{3+}$ and $\mathrm{Fe}^{2+}$ ions. ${ }^{32} \mathrm{Up}$ to the maximum pressure of $65 \mathrm{GPa}$, the unchanged energy position of the pre-peak indicates no valence change of A-site $\mathrm{Fe}^{3+}$ (Fig.1c inset), which rules out a transition from the inverse to normal spinel $\mathrm{Fe}_{3} \mathrm{O}_{4}$ under pressure as proposed in ref. ${ }^{7,16-19}$

The pressure induced changes of energy position of the main peak $\beta\left(\mathrm{E}_{\beta}\right)$ (whiteline) 

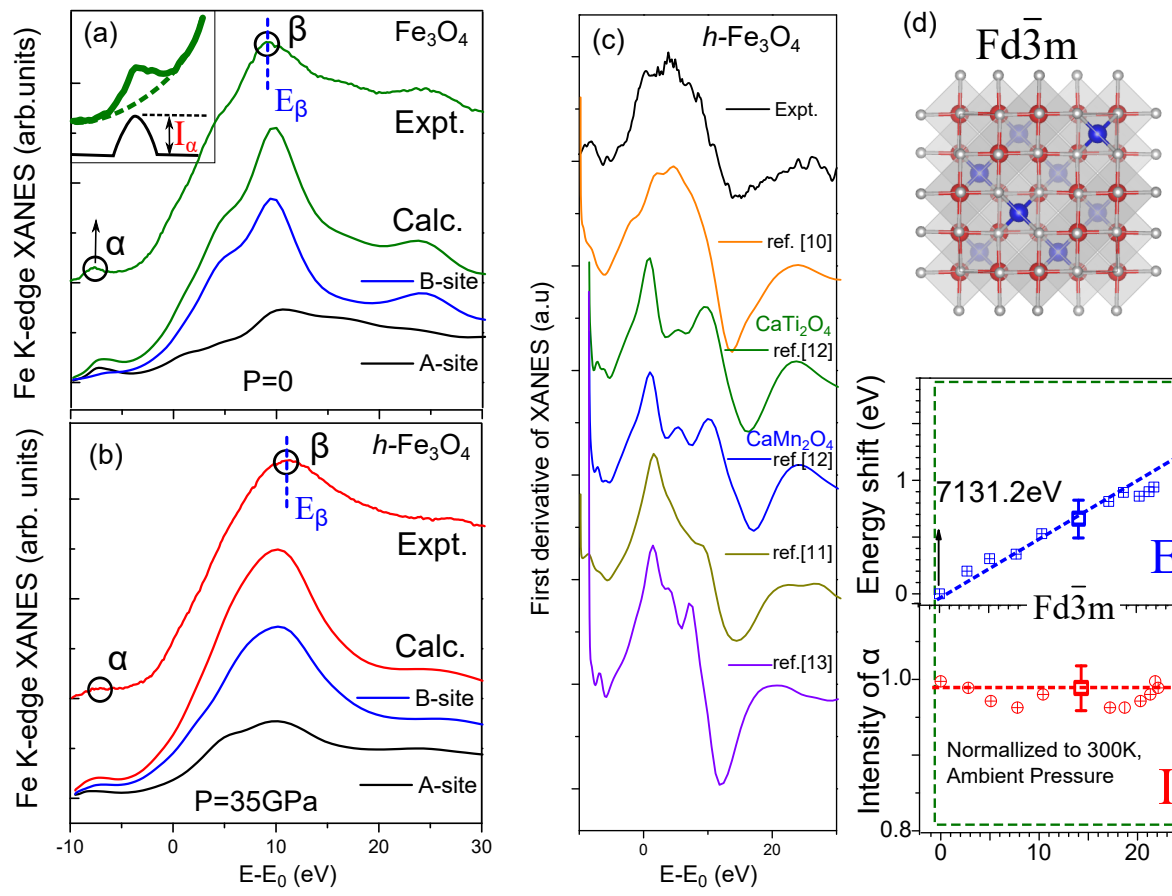

(e) $\mathrm{Bbmm}$
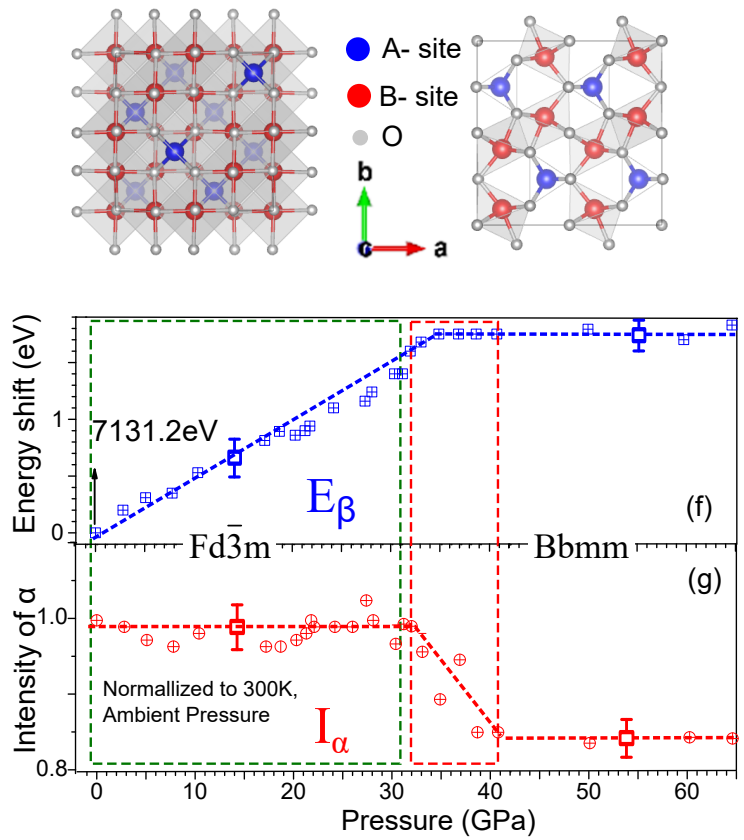

Figure 2: Pressure dependent XANES of $\mathrm{Fe}_{3} \mathrm{O}_{4}$. Experimental and calculated XANES spectra with separated A (black line) and $\mathrm{B}$ site contributions (blue line) of $\mathrm{Fe}_{3} \mathrm{O}_{4}$ at (a) $\mathrm{P}=0$ and (b) $\mathrm{P}=35 \mathrm{GPa}$. The $(\mathrm{d})$ magnetite structure $(\mathrm{Fd} \overline{3} \mathrm{~m})$ and $(\mathrm{e}) h-\mathrm{Fe}_{3} \mathrm{O}_{4}$ with $\mathrm{CaTi}_{2} \mathrm{O}_{4}$-type $(\mathrm{Bbmm})^{10}$ are used for the calculation of $\mathrm{P}=0$ and $\mathrm{P}=35 \mathrm{GPa}$, respectively. First derivative of the experimental and theoretical simulated XANES, using the structure from ref., ${ }^{10-13}$ are listed in (c). Defined from the XANES, the pressure induced changes of the energy of the main peak $\beta\left(\mathrm{E}_{\beta}\right)$ and the intensity of the pre-peak $\alpha\left(\mathrm{I}_{\alpha}\right.$, defned in the inset of Fig.1a) are shown in (f) and (g), indicates that the $h-\mathrm{Fe}_{3} \mathrm{O}_{4}$ starts to appear at $\mathrm{P} \sim 30 \mathrm{GPa}$. The error bars represent the standard deviation of the value from data evaluation.

and the normalized intensity (according to the intensity at 300K, ambient pressure) of the pre-peak $\alpha\left(\mathrm{I}_{\alpha}\right.$, defined as the height of peak $\alpha$ in Fig.2a inset) are shown in Fig. 2e and 2f. Unlike pre-edge peak $\alpha$ that is sensitive to local symmetry, the profile of the main peak $\beta$ is sensitive to both local and long range crystal structure. According to calculations (see Fig.S2 in supplementary materials), without structural change the pressure induced lattice compression will only give energy shifts for XAS profile due to the reduction of the Fe-O bonds, while the pre-edge peak position, intensity and the XANES shape remain unchanged, as we observed for $\mathrm{P}<27.4 \mathrm{GPa}$. The reduced $\mathrm{I}_{\alpha}$ and the change of spectral profile of the main peak $\beta$ at $\mathrm{P}>27.4 \mathrm{GPa}$ indicate the formation of a new lattice structure, that is, the high pressure phase $h$ - $\mathrm{Fe}_{3} \mathrm{O}_{4}$. According to x-ray diffraction results, the $h-\mathrm{Fe}_{3} \mathrm{O}_{4}$ may 
have the structure of $\mathrm{CaTi}_{2} \mathrm{O}_{4}$-type $(\mathrm{Bbmm})^{10-12}$ or $\mathrm{CaMn}_{2} \mathrm{O}_{4}$-type $(\mathrm{Pbcm}) .{ }^{9}$ Above $40 \mathrm{GPa}$ $\mathrm{I}_{\alpha}$ remains nearly unchanged and the energy position of the main peak $\beta$ exhibits a weak energy shift as a function of pressure as shown in Fig.1e, since $h$ - $\mathrm{Fe}_{3} \mathrm{O}_{4}$ has a compact crystal structure $^{9-12}$ compared to magnetite $(\mathrm{Fd} \overline{3} \mathrm{~m})$, it exhibites a weak pressure response.

In these proposed structural models ${ }^{9,11,12}$ all $\mathrm{Fe}^{3+}$ ions are located on B-sites (8f site) with an octahedral coordination, while the $\mathrm{Fe}^{2+}$ ions are located on the A-sites (4c site) with an eightfold coordination (bi-capped trigonal prismatic) and much longer Fe-O bonds than in the B-sites. Our calculations shows that the XANES of such structures for $h-\mathrm{Fe}_{3} \mathrm{O}_{4}$ with a charge transfer between B-sites and A-sites, have very different spectrum profile compared to the experimental spectrum (Fig.S3 in supplementary). We also checked the most recent proposed crystal structure from single crystal XRD, ${ }^{13}$ the simulated XANES comes near to the experimental results, except for a weak shoulder peak (marked in Fig.S3f in the supplementary), due to the similar lower valence of A-site Fe ions in this structure. Our calculated XANES spectra are, on the other hand, in fairly good agreement with the experimental XANES spectrum, if a Bbmm structure with the $\mathrm{Fe}^{2+}$ ions located in the Bsites, is considered. Please see Fig. 2a and 2b, where the experimental XANES spectra of $\mathrm{Fe}_{3} \mathrm{O}_{4}$ at $\mathrm{P}=0$ and $35 \mathrm{GPa}$ are shown together with the calculated XANES. The separated A- (black line) and B-sites (blue line) contributions are also reported. In Fig.2c, the first derivative of the experimental and theoretical simulated XANES, using the structure from ref., ${ }^{10-13}$ are listed for comparison. Here, the experimental results can only be well simulated with the $\mathrm{CaTi}_{2} \mathrm{O}_{4}$ structure reported in ref. ${ }^{10}$ Our data analysis therefore supports the $\mathrm{CaTi}_{2} \mathrm{O}_{4}$ structure of $h-\mathrm{Fe}_{3} \mathrm{O}_{4}$. Besides, the valence of Fe ions were confirmed to be $3+$ at Asites (4c site) and mixed of $2+$ and $3+$ at B-sites (8f site) in the $h-\mathrm{Fe}_{3} \mathrm{O}_{4}$ phase, respectively. Thus we rule out the charge transfer between B-sites and A-sites Fe ions through the structure transition.

Having established the coordination of the $\mathrm{Fe}^{2+}$ and $\mathrm{Fe}^{3+}$ ions, as second step we evaluated the local structure by exploiting the high sensitivity of XANES to the Fe-O bond-length 

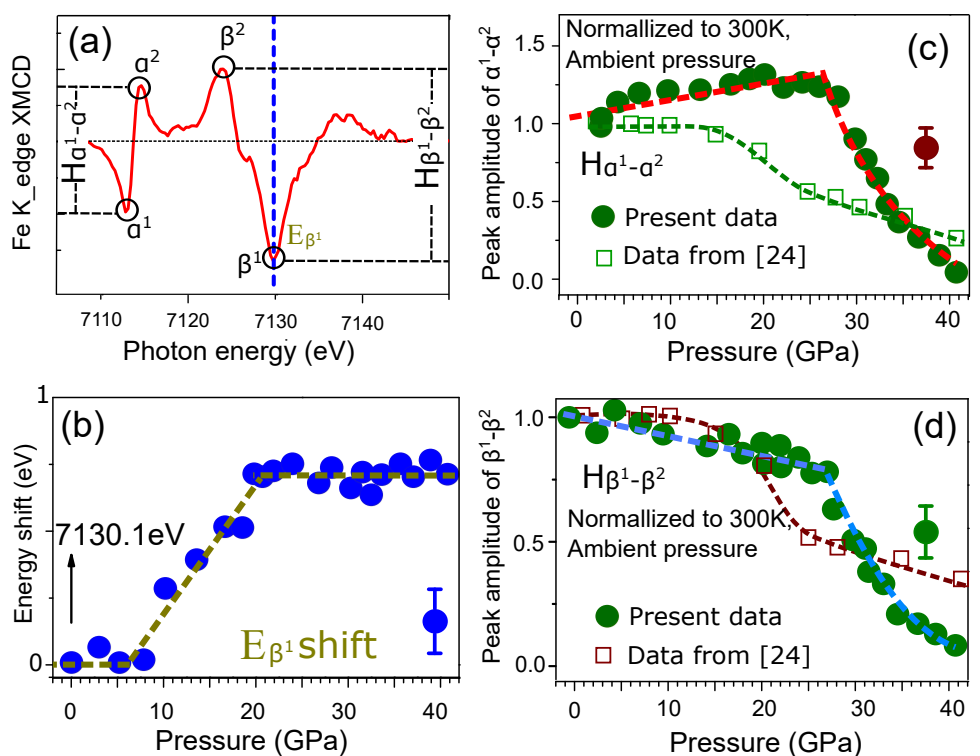

Figure 3: Pressure induced changes of the XMCD spectra of $\mathrm{Fe}_{3} \mathrm{O}_{4}$. Defined from the XMCD (a), the pressure induced changes of peak $\beta^{1}$ energy $\left(\mathrm{E}_{\beta^{1}}\right)$, and the intensities of the XMCD peaks $\left(H_{\alpha^{1}-\alpha^{2}}\right.$ and $H_{\beta^{1}-\beta^{2}}$ ), representing the absolute height from $\alpha^{1}$ to $\alpha^{2}$ and from $\beta^{1}$ to $\beta^{2}$, are shown in (b), (c) and (d) with the error bars, and the XMCD intensity extracted from ref. ${ }^{24}$ are also shown for comparison. The intensity of XMCD starts to decrease at $\sim 27.4 \mathrm{GPa}$ when entering the $h$ - $\mathrm{Fe}_{3} \mathrm{O}_{4}$ for the present data. The error bars represent the standard deviation of the value from data evaluation.

distribution. No good fit of the XANES spectra could be obtained in the case of the heavily distorted cation environment of the $\mathrm{CaMn}_{2} \mathrm{O}_{4}$-type structure $(\mathrm{Pbcm})$. Using the oxygen position of the Bbmm structure proposed in, ${ }^{11,12}$ where the A-site (B-site) Fe-O bond-lengths are relatively long (short), provides a theoretical XANES that is also someway different from the experimental one (see Table. S3-5 and Fig.S3 in supplementary materials). A fairly nice simulation (Fig. 2b) of the experimental spectra was obtained for the Bbmm structure when using the oxygen positions given by Haavik et al. ${ }^{10}$ (see Table.S2 in supplementary materials). No evidence of charge transfer or spin transition was observed in $h-\mathrm{Fe}_{3} \mathrm{O}_{4}$ since the XANES spectra keep unchanged up to 65 GPa at 300K (see Fig. 1e).

Pressure response of XMCD signal $\alpha^{1}-\alpha^{2}$ and $\beta^{1}-\beta^{2}$, normalized to the intensity at 300K and ambient pressure, are listed in Fig. 3. Slight changes of the peak height of $\alpha^{1}-\alpha^{2}$ and $\beta^{1}-\beta^{2}$, defined in Fig. 3a as $H_{\alpha^{1}-\alpha^{2}}$ and $H_{\beta^{1}-\beta^{2}}$, respectively, have been observed from 0 to $27.4 \mathrm{GPa}$ followed by a rapid decrease to 0 from 27.4 to $40 \mathrm{GPa}$. Here, no abrupt change 


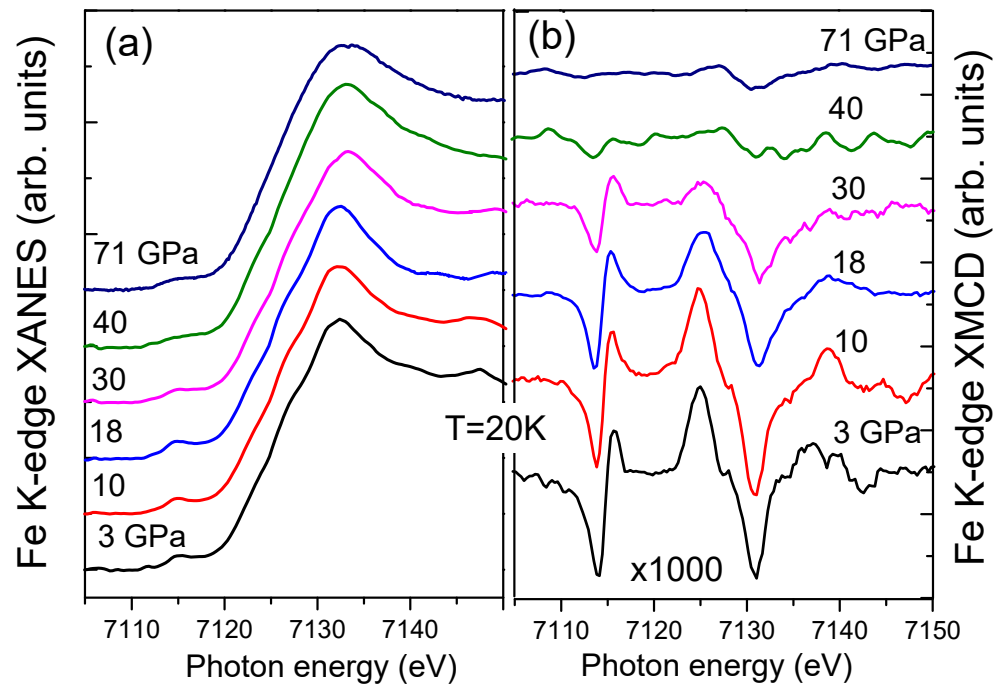

Figure 4: Pressure dependent Fe K-edge XANES and XMCD spectra at $\mathrm{T}=20 \mathrm{~K}$. $\mathrm{Fe}_{3} \mathrm{O}_{4}$ enters into the $h-\mathrm{Fe}_{3} \mathrm{O}_{4}$ phase with $\mathrm{P}>30 \mathrm{GPa}$ with the reduced pre-peak $\alpha$ in XANES (a) and disappeared XMCD (b) signal, $H_{\alpha^{1}-\alpha^{2}}$ and $H_{\beta^{1}-\beta^{2}}$ at $20 \mathrm{~K}$.

of XMCD signal $\left(H_{\alpha^{1}-\alpha^{2}}\right.$ and $\left.H_{\beta^{1}-\beta^{2}}\right)$ was observed up to $\mathrm{P}=27.4 \mathrm{GPa}$ from our results as shown in Fig. 3c and 3d ruling out the sharp spin state transition of B-site $\mathrm{Fe}^{2+}$ proposed in Ref. ${ }^{14}$ Our results are in agreement with very recent high pressure experimental $\mathrm{Fe} \mathrm{K}_{\beta} \mathrm{X}$-ray emission spectra on $\mathrm{Fe}_{3} \mathrm{O}_{4},{ }^{20}$ which showed no loss of local spin moment under pressure below $20 \mathrm{GPa}$ and excluded the HS $\rightarrow$ IS or HS $\rightarrow$ LS transition. The energy shift of the XMCD peak $\beta^{1}\left(\mathrm{E}_{\beta^{1}}\right.$, Fig. 3b) is related to the pressure induced energy shift of the XANES main peak $\beta$ $\left(\mathrm{E}_{\beta}\right)$ resulting from the shorten Fe-O bonding length. Here we see a rapid decrease of $H_{\alpha^{1}-\alpha^{2}}$ and $H_{\beta^{1}-\beta^{2}}$ from $27 \mathrm{GPa}$ to $40 \mathrm{GPa}$. The disappearance of XMCD signal indicates the loss of net ordered magnetic moment in $h-\mathrm{Fe}_{3} \mathrm{O}_{4}$ phase.

The pressure induced phase transition of $\mathrm{Fe}_{3} \mathrm{O}_{4}$ was considered to be sensitive to the hydrostatic conditions when different PTMs are used. However, our XANES and XMCD results of $\mathrm{Fe}_{3} \mathrm{O}_{4}$ are the same when different PTMs of silicon oil, $\mathrm{O}_{2}$ or Ne were used up to $65 \mathrm{GPa}$ at $300 \mathrm{~K}$. Besides, the phase transition are more sensitive to the smaple quality under investigation. In Fig.3c and 3d, the XMCD intensity extracted from ref. ${ }^{24}$ are listed for comparison. For the micrometer-sized magnetite commercial powder with broad Verwey transition, the phase transition from magnetite $\mathrm{Fe}_{3} \mathrm{O}_{4}$ to $h-\mathrm{Fe}_{3} \mathrm{O}_{4}$ may also be observed in a 
broad pressure range. In ref. ${ }^{24}$ the phase transition starts at 10-15 GPa and not completed up to $41 \mathrm{GPa}$, resulting in the decrease of XMCD intensity from 10-15 GPa and non-zero XMCD intensity up to $41 \mathrm{GPa}$. A much sharp phase transition from 27-35 GPa has been observed according to the XMCD intensity of present data.

There are four possible scenarios to explain the loss of XMCD signal: (1) $h-\mathrm{Fe}_{3} \mathrm{O}_{4}$ phase is paramagnetic as the ordering temperature drops, (2) the so-called magnetic collapse into a nonmagnetic metallic state, (3) the complete suppression of the magnetic order due to magnetic frustration or (4) the formation of antiferromagentic order in the new structure. Pressure dependent XANES and XMCD spectra at $\mathrm{T}=20 \mathrm{~K}$ were measured and shown in Fig. 4 (a) and (b), respectively. Similar to room temperature, $h-\mathrm{Fe}_{3} \mathrm{O}_{4}$ is formed at around 3040 GPa. Disappearance of XMCD signal at low-temperature $(\mathrm{T}=20 \mathrm{~K})$ under high-pressure $(\mathrm{P}=40,71 \mathrm{GPa})$ demonstrate that a decrease of $\mathrm{T}_{c}$ below room temperature under high pressure can be ruled out. Although the previous resistivity measurements had shown controversial results, ${ }^{27,28}$ neither of them show any sharp drop of resistivity in $h$ - $\mathrm{Fe}_{3} \mathrm{O}_{4}$ phase, therefore we can excluded the scenario of magnetic collapse.

The scenario of structure change induced magnetic frustration or antiferromagentic ordering can not be excluded here. The first principle calculations ${ }^{33}$ pointed out that the strong antiferromagnetic coupling $\left(\mathrm{J}_{A-B}\right)$ between the A- and B-sites Fe ions dominates in magnetite $\mathrm{Fe}_{3} \mathrm{O}_{4}$. Entering the $h-\mathrm{Fe}_{3} \mathrm{O}_{4}$, the antiferromagnetic exchange coupling between A- and B-sites Fe ions, may get depressed. Besides, in the $h-\mathrm{Fe}_{3} \mathrm{O}_{4}$ the A-sites Fe ions form chains along the c-axis and the AFM coupling between the A-sites is not anymore weak but probably of the same order as the AFM coupling between the A and B sites. Such a change of the dominant magnetic coupling might lead to the magnetic frustration in A- and B-sites Fe ions losing net magnetic moment in $h-\mathrm{Fe}_{3} \mathrm{O}_{4}$. Even a pure AFM order ${ }^{13}$ with all spins coupled antiparallel along the c-axis may form if AFM $\mathrm{J}_{A-A}$ is strong enough in $h-\mathrm{Fe}_{3} \mathrm{O}_{4}$. In both cases the XMCD signal would be very small for a small applied field as $\mu_{0} \mathrm{H}=1.3 \mathrm{~T}$ used in our measurements. Further first principle calculations ${ }^{33}$ are desirable to confirm whether 
these scenarios are the cause of the loss of the net ordered moment in $h-\mathrm{Fe}_{3} \mathrm{O}_{4}$. According to the Mössbauer spectroscopy, ${ }^{13}$ the HS-LS transition scenario of B-site $\mathrm{Fe}^{3+}$ in $h$-Fe ${ }_{3} \mathrm{O}_{4}$ was suggested to start at $50 \mathrm{GPa}$, however, no evidence of the transition was observed from the present XANES and XMCD results.

To conclude, phase transitions of $\mathrm{Fe}_{3} \mathrm{O}_{4}$ have been revisited by performing Fe K-edge $\mathrm{XAS}$ and XMCD measurements under pressure. The comparison of the XANES spectra measured at P>30 GPa, with theoretical simulations from MXAN indicates that the high pressure phase $h-\mathrm{Fe}_{3} \mathrm{O}_{4}$ has a $\mathrm{CaTi}_{2} \mathrm{O}_{4}(\mathrm{Bbmm})$ structure. The drop in XMCD intensity of $h$ $\mathrm{Fe}_{3} \mathrm{O}_{4}$ interpreted in terms of a complete suppression of the magnetic order due to the strong magnetic frustration or antiferromagnetic ordering. Both the scenarios of spin-crossover and charge transfer have been deterministically ruled out. Mössbauer spectroscopy investigation ${ }^{13}$ proposed second order high to low spin transition in B-site $\mathrm{Fe}^{3+}$ of $h$ - $\mathrm{Fe}_{3} \mathrm{O}_{4}$ from 50 to $80 \mathrm{GPa}$ but this statement requires further confirmation form XANES or Fe $\mathrm{K}_{\beta}$-resonant $\mathrm{X}$-ray emission spectra, which is more sensitive to pressure induced spin transition. ${ }^{20,34,35}$

The authors thank SOLEIL for provision of synchrotron radiation facilities.

\section{References}

(1) Weiss, P.; and Forrer, R.; La saturation absolue des ferromagnétiques et les lois d'approche en fonction du champ et de la température. Ann. Phys. (Paris) 1929, 12, 279.

(2) Verwey, E. J. W.; Electronic Conduction of Magnetite $\left(\mathrm{Fe}_{3} \mathrm{O}_{4}\right)$ and its Transition Point at Low Temperatures. Nature (London) 1939, 144, 327.

(3) Walz, F.; The Verwey transition - a topical review. J. Phys. Condens. Matter 2002, 14, R285.

(4) Garcia, J.; and Subias, G.; The Verwey transition-a new perspective. J. Phys. Condens. Matter 2004 16, R145. 
(5) Wright, J.P.; Attfield, J.P. and Radaelli, P.G.; Long Range Charge Ordering in Magnetite Below the Verwey Transition. Phys. Rev. Lett.2001 87, 266401; Charge ordered structure of magnetite $\mathrm{Fe}_{3} \mathrm{O}_{4}$ below the Verwey transition. Phys. Rev. B 2002 66, 214422.

(6) Senn, M.S.; Wright, J.P. and Attfield, J.P.; Charge order and three-site distortions in the Verwey structure of magnetite, Nature 2012 481, 173.

(7) Rozenberg, G.Kh.; Pasternak, M.P.; Xu, W.M.; et al.; Origin of the Verwey Transition in Magnetite, Phys. Rev. Lett. 2006 96, 045705.

(8) Mao, H.-K.; Takahashi, T.; Bassett, W.; et al.; Isothermal compression of magnetite to 320 KB, J. Geophys. Res. 1974 79, 1165.

(9) Fei, Y.; Frost, D.J.; Mao, H.-K.; et al.; In situ structure determination of the high-pressure phase of $\mathrm{Fe}_{3} \mathrm{O}_{4}$, Am. Mineral. 1999 84, 203.

(10) Haavik, C.; Stolen, S.; Fjellvag, H.; et al.; Equation of state of magnetite and its high-pressure modification: Thermodynamics of the Fe-O system at high pressure, Am. Mineral. 2000 85, 514.

(11) Dubrovinsky, L.S.; Dubrovinskaia, N.A.; McCammon, C.; et al.; The structure of the metallic high-pressure $\mathrm{Fe}_{3} \mathrm{O}_{4}$ polymorph: experimental and theoretical study, J. Phys.: Condens. Matter 2003 15, 7697-7706.

(12) Ricolleau, A.; Fei, Y.;Equation of state of the high-pressure Fe3O4 phase and a new structural transition at $70 \mathrm{GPa}$, Am. Mineral. 2016 101, 719.

(13) Greenberg, E.; Xu, W.M.; Nikolaevsky, M.; et al.; High-pressure magnetic, electronic, and structural properties of $\mathrm{MFe}_{2} \mathrm{O}_{4}(\mathrm{M}=\mathrm{Mg}, \mathrm{Zn}, \mathrm{Fe})$ ferric spinels, Phys. Rev. B 2017 95, 195150.

(14) Ding, Y.; Haskel, D.; Ovchinnikov, S.G.; et al.; Novel Pressure-Induced Magnetic Transition in Magnetite $\left(\mathrm{Fe}_{3} \mathrm{O}_{4}\right)$ Phys. Rev. Lett. 2008 100, 045508. 
(15) Ovsyannikov, S.V.; Shchennikov,V.V.; Todo, S.; Uwatoko, Y.; A new crossover in $\mathrm{Fe}_{3} \mathrm{O}_{4}$ magnetite under pressure near $6 \mathrm{GPa}$ : modification to 'ideal' inverse cubic spinel?, J. Phys. Condens. Matter. 2008 20, 172201.

(16) Rozenberg, G.Kh.; Hearne, G.R.; and Pasternak, M. P.; Nature of the Verwey transition in magnetite $\left(\mathrm{Fe}_{3} \mathrm{O}_{4}\right)$ to pressures of $16 \mathrm{GPa}$, Phys. Rev. B 1996 53, 6482.

(17) Todo, S.; Takeshita, N.; Kanehara, K.; Mori, T.; and Môri, M.; Metallization of magnetite $\left(\mathrm{Fe}_{3} \mathrm{O}_{4}\right)$ under high pressure J. Appl. Phys. 2001 89, 7347.

(18) Pasternak, M.P.; Xu, W.M.; Rozenberg, G.Kh.et al.; Pressure-induced coordination crossover in magnetite; the breakdown of the Verwey-Mott localization hypothesis J. Magn. Magn. Mater. 2003265, L107.

(19) Rozenberg, G.Kh.; Amiel, Y.; Xu, W.M.; et al.; Structural characterization of temperatureand pressure-induced inverse-normal spinel transformation in magnetite, Phys. Rev. B 2007 $75,020102$.

(20) Lin, J-F.; Wu, J.; Zhu, J.; et al.; Abnormal elastic and vibrational behaviors of magnetite at high pressures, Sci. Rep. 2014 4, 6282.

(21) Klotz, S.; Steinle-Neumann,G.; Strässle, Th.; et al.; Magnetism and the Verwey transition in $\mathrm{Fe}_{3} \mathrm{O}_{4}$ under pressure, Phys. Rev. B 2008 77, 012411.

(22) Gatta, G. D.; Kantor, I.; Ballaran, T.B.; et al.; Effect of non-hydrostatic conditions on the elastic behaviour of magnetite: An in situ single-crystal X-ray diffraction study, Phys. Chem. Miner. 2007 34, 627.

(23) Subias, G.; Cuartero, V.; Garcia, J.; et al.; Pressure-induced magnetic transition in $\mathrm{Fe}_{3} \mathrm{O}_{4}$ and $\mathrm{CoFe}_{2} \mathrm{O}_{4}$ spinels, J. Phys.: Conf. Ser. 2009 190, 012089.

(24) Baudelet, F.; Pascarelli, S.; Mathon, O.; et al.; Absence of abrupt pressure-induced magnetic transitions in magnetite, Phys. Rev. B 2010 82, 140412(R). 
(25) Ju, S.; Cai, T-Y.; Lu, H.-S.; and Gong, C-D.; Pressure-Induced Crystal Structure and SpinState Transitions in Magnetite $\left(\mathrm{Fe}_{3} \mathrm{O}_{4}\right)$ J. Am. Chem. Soc. 2012 134, 13780-13786.

(26) Bengtson, A.; Morgan, D.; Becker, U.; Spin state of iron in $\mathrm{Fe}_{3} \mathrm{O}_{4}$ magnetite and h- $\mathrm{Fe}_{3} \mathrm{O}_{4}$, Phys. Rev. B 2013 87, 155141.

(27) Xu, W.M.; Machavariani, G.Y.; Rozenberg, G.K.; Pasternak, M.P.; Mössbauer and resistivity studies of the magnetic and electronic properties of the high-pressure phase of $\mathrm{Fe}_{3} \mathrm{O}_{4}$, Phys. Rev. B 2004 70, 174106.

(28) Muramatsu, T.; Gasparov, L.V.; Berger, H.; Hemley, R.lJ.; Struzhkin, V.V.; Electrical resistance of single-crystal magnetite $\left(\mathrm{Fe}_{3} \mathrm{O}_{4}\right)$ under quasi-hydrostatic pressures up to $100 \mathrm{GPa}$, J. Appl. Phys. 2016 119, 135903.

(29) Baudelet, F.; Nataf, L.; Torchio, R.; New scientific opportunities for high pressure research by energy-dispersive XMCD, High Pres. Res., 2016 36(3): 429 444.

(30) Benfatto, M.; Congiu-Castellano, A.; Daneile, A.; Longa, S.D.; MXAN : a new software procedure to perform geometrical fitting of experimental XANES spectra, J. Synchrotron Radiat, $20018,267-269$.

(31) Schütz, G.; Wagner, W.; Wilhelm, W.; et al.; Absorption of circularly polarized x rays in iron, Phys. Rev. Lett. 1987 58, 737.

(32) Arrio, M.-A.; Rossano, S.; Brouder, Ch.; Galoisy, L.; Calas, G.; Calculation of multipole transitions at the Fe K pre-edge through p-d hybridization in the Ligand Field Multiplet model, Europhys. Lett. 2000 51, 454.

(33) Leonov, I.; Yaresko, A.N.; Antonov, V.N.; Anisimov, V.I.; Electronic structure of chargeordered $\mathrm{Fe}_{3} \mathrm{O}_{4}$ from calculated optical, magneto-optical Kerr effect, and O K-edge x-ray absorption spectra, Phys. Rev. B 2006 74, 165117.

(34) Rueff, J.-P.; Shukla, A.; Inelastic x-ray scattering by electronic excitations under high pressure, Rev. Mod. Phys. 2010 82, 847. 
(35) Shen, G.; Mao, H.-K.; High-pressure studies with x-rays using diamond anvil cells, Rep. Prog. Phys. 2017 80, 016101. 